\title{
Lindes fenomenológicos de la psiquiatría existencial.
}

Phenomenological boundaries of existential psychiatry.

\author{
Sergi Solé Plans a.
}

${ }^{a}$ Psiquiatra, Centro de Salud Mental de Adultos Horta, Barcelona.

Correspondencia: Sergi Solé Plans (soleplans@gmail.com)

Recibido: 02/03/2011; aceptado: 04/07/2011

RESUMEN: La psiquiatría existencial debería apartarse de la filosofía de la conciencia para articular una relación terapéutica y no sólo de conocimiento. Sin embargo, el método fenomenológico que se proponía auxiliarla permanece en el saber/ conocer sin dar cuenta del saber/hacer del estar en relación. Esta sumisión a la conciencia se descubre en el Análisis Existencial contemporáneo a través de la psicopatología de un sujeto escindido sin esperanzas de curación. En otra lectura enterada de sus límites metodológicos, la fenomenología se ciñe a la tarea de despejar un lugar de encuentro. Tomando la dirección de la filosofía de la existencia, inaugurada por Søren A. Kierkegaard y culminada en el Heidegger tardío, ofrece un camino posible por el que establecer una relación más allá del conocer.

PALABRAS CLAVE: Psiquiatría, Existencialismo, Psicología existencial, Psicopatología, Psicoterapia.

\begin{abstract}
Existential psychiatry should draw itself away from the philosophy of conscience so as to articulate not only a knowledge-based relationship but also a therapeutic one. Nonetheless, the phenomenological method which was ment to assist it lies in knowledge as understanding and does not give account for knowledge as action of being in relationship. This submission to conscience is uncovered in contemporary Existential Analysis through the psychopathology of a split subject beyond hope of cure. In another reading aware of its methodological limits, phenomenology keeps to the task of clearing a meeting place. Following the directions of the philosophy of existence, founded by Søren A. Kierkegaard and culminated in late Heidegger, it offers a possible way to establish a relationship beyond knowledge.

KEYWORDS: Psychiatry, Existentialism, Existential Psychology, Psychopathology, Psychotherapy.
\end{abstract}

\section{Introducción}

La corriente existencial viene presentándose en psiquiatría como una ansiada tercera opción entre las discordias imperantes. Mediando las diatribas del psicoanálisis con el objetivismo de Heidelberg o el conductismo skinneriano, intentó abrirse paso en la psiquiatría europea y la psicología estadounidense $(1,2)$. Popularmente identificada con el movimiento contracultural de los años sesenta, desde el flower power yanqui al mayo del 68 francés, ha primado de ella una lectura sociocultural polarizada ante la controvertida irracionalidad (3). Si al otro lado del Atlántico la corriente existencial se diluía (entre neofreudianos y gestaltistas) en un humanismo de compleja definición (4), se fundió en el viejo continente sin ambages con la empresa fenomenológica. Y si en la aventura americana su conceptualización se torna 
laberíntica, en Europa se ve claramente sometida a los términos prestados por el método fenomenológico, sin llegar a quedar claro nunca cuáles son sus fundamentos propios ni qué tiene de suyo, esto es, de existencial.

En el presente trabajo postulamos que la psiquiatría existencial no ha tomado apenas nada de la filosofía homónima. De algún modo ha parecido no bastar a los terapeutas "existenciales" el sostén de la filosofía de la existencia. Tal vez el temor a un pensamiento no categorizado, escasamente protocolizable y entregado a burdas críticas de acientificismo, los ha compelido a retomar el asidero fenomenológico. La psiquiatría existencial viene sufriendo así una pasmosa bicefalia "fenomenológico-existencial" sin aclarar la necesidad de unos cimientos tan compulsivamente asegurados.

Defendemos que el análisis existencial (nombre dado a la práctica existencial en psiquiatría fundada por Ludwig Binswanger) se ha ceñido a una lectura temprana (cuando no viciada) de la obra de Edmund Husserl; y ha descuidado, salvada la apariencia, las transformaciones que justificaran una proximidad más que nominal respecto a la analítica existencial de Martin Heidegger. Intentaremos ilustrar los límites -y escasa problematización- de esa lectura en el análisis existencial contemporáneo, especialmente a través de algunos de sus actuales representantes en la International Federation of Daseinsanalysis.

Veremos qué posibilidades nos ofrecen y cuáles nos escatiman los paradigmas estudiados, fenomenológico y existencial. Para ello analizaremos los matices que aportan al concepto verdad, y a su luz el de sujeto, ya que la diferente intelección del primero condicionará el modo de comprender a la persona: "Lo que pone en marcha el movimiento que conduce a la tesis moderna de la subjetividad es la transformación de la verdad en certeza" afirma Ramón Rodríguez (5). Si queremos presentar al trato médico un sujeto distinto del moderno, no expuesto a la disección encefálica ni a la interpretación libidinal, deberemos proceder a la reconceptualización de la verdad. Este será el marco de discusión en el que pretendemos dibujar un nuevo escenario para el trato. No basta renombrar (con el ya insufrible matraqueo de la angustia, la autenticidad, la libertad, la finitud y la muerte) mecanismos asimilados de otros modelos, como la versión dinámica existencial de Irvin Yalom (6). Hay que alumbrar una distinción más radical: la nueva comprensión del ser como posible, distinto del artificioso doblete empírico-trascendental ingeniado por la metafísica de la subjetividad, o de la certeza.

Si la condición trascendental resulta aceptablemente clara en los supuestos del modelo anatomo-clínico y de la metapsicología freudiana, cerebro y aparato psíquico, han permanecido veladas las implicaciones del sujeto de la conciencia emanado de una intelección fenomenológica en psiquiatría. Por tanto, si una crítica nos parece necesaria es la de este ladino cognoscente perturbado que lastró los desarrollos existenciales de nuestra disciplina. 
Habrá que ver si el sujeto fenomenológico se desmarca o sucumbe a aquellas prácticas de certeza objetivante sujetas -como señala Antonio Diéguez- "a una visión de la realidad que plantea como posible y deseable alcanzar la verdad" (7), e incluso partir de ella. Cabrá dilucidar si su verdad responde a la teoría tradicional del conocimiento o si se aproxima a una corriente existencial que, entendiendo la verdad como proceso y no como fin o principio, ve despejado el camino hacia el preguntar fundamental que pretende. $\mathrm{Si}$, ajeno a precipitadas ecuaciones entre lo útil, la verdad y la esencia, deja abierto un discurso que se muestre vivo en cada una de las existencias concretas que lo constituyen, en cada uno de los dolientes que lo solicitan.

\section{¿Por qué no somos posmodernos?}

El vagar del pensamiento ha querido hermanar la perspectiva existencial con el relativismo constructivista posmoderno y sus terapias narrativas (8). Lejos del desaire a la verdad que éstas exhiben, la filosofía de la existencia no sólo reconoce sino que parte de su condición epistémica, si bien por su reverso, y concibe así una teoría de lo humano (de la verdad) incompatible con la celebrada integración. Se aparta de las tentativas posmodernas que, desdeñando la verdad, se rinden inadvertidamente a ella. Pues aunque se aspavienten ante el declarado anhelo positivista por una verdad absoluta, su propuesta no se distingue tanto si no es por el sigilo en su proceder (9) ${ }^{1}$. Mientras unos pretenden abiertamente alcanzar las verdades trascendentes, los otros se aplican con disimulo en la construcción de las mismas. Acción, producción y creatividad de la verdad son proclamas que desde el constructivismo terapéutico posmoderno no abandonan la creencia en una verdad accesible y proponen -siguiendo a Richard Rorty- narrativas transformadoras de una verdad despreciada en favor de otra. La psiquiatría existencial debe rechazar el afán constructivista rortyano por "re-imaginarnos en nuevas personas" (7), pues apela a la persona que ya es. Se aparta del combate narrativo, de la pugna dialéctica. No cree que la mutación del relato construya una nueva realidad, ya que como advierte Heidegger "no debe decirse del logos que es el lugar primario de la verdad" (10). El alemán ofrece el lenguaje como morada del ser, como marco deslindado; condiciona el sentido del ser a la palabra sin identificarlo con ella. Rechaza el encorsetamiento romano del hombre como animal rationale. La verdad está ya en el ser y, por tanto, la tarea terapéutica consistirá no en proponerla o imponerla (como

\footnotetext{
${ }^{1}$ Goldenberg H. Fact or Fiction? The Relationship of 'Fact' and 'Narrative'. En Du Plock (9), pp. 306-17
} 
los posmodernos, aunque tampoco en subponerla como los modernos hacían) sino en descubrirla, en simplemente mostrarla, o ni siquiera: en dejar que la persona la muestre. "Como médico uno debe, por decirlo así, recogerse y permitir que la otra persona sea", sugiere Martin Heidegger en los Seminarios de Zollikon ${ }^{2}$ (11). Mas para habitar este escenario abierto a un aparecer y un mostrarse "es de fundamental importancia -advierte el de Messkirch- liberarse de un concepto constructivo de la verdad que la entiende como «concordancia»" (10). No se trata de que nuestro discurso alcahuetee narrativas y realidades, de que se enteste en su forzada unión. Hay que despejar un espacio en el que la persona pueda libremente exponerse, explicarse. Su verdad, lejos de ser pretendida o desdeñada, debe ser recomprendida.

\section{Qué decepción cuando es lo que parece}

Si apuntábamos en la introducción que el avance de la psiquiatría existencial se ha visto obstaculizado por un excesivo compromiso con los supuestos fenomenológicos, el relativismo rortyano (12) que acabamos de censurar florece tras la pérdida de éstos. La propuesta existencial deberá situarse -de nuevo- en una posición mediadora. Ni anclada en la fenomenología ni abandonada a la suerte constructivista.

Sea entendida la analítica existencial de Martin Heidegger como superación, transformación o destilación de la fenomenología de Edmund Husserl, interesa aquí destacar una evolución que no parece haber atendido el análisis existencial contemporáneo. Varado en los primeros desarrollos de la fenomenología husserliana, preso de una tradición fundada en la primacía del conocimiento, le resulta ímproba la tarea por la que abandonar la antigua metafísica de la subjetividad, la conocida historia de sujeto-conoce-objeto. El primer lance de la empresa existencial lo data Paul Tillich (historiador de cabecera de la psicología existencial americana) en la década de 1840 (13). La crítica al racionalismo hegeliano trata entonces de rechazar la identificación entre realidad y razón, entre la realidad y su conocimiento. Søren Kierkegaard, padre de la filosofía de la existencia, protagonizará la ruptura de ese esquema metafísico hegeliano para fundar un nuevo modo de estar en el mundo. No será ya cuestión de hurgar en la relación entre los supuestos del saber, objeto conocido y sujeto cognoscente; porque saber ya no será sólo conocer,

\footnotetext{
2 Conocidos por este nombre -literalmente traducido del alemán Zollikoner Seminare, y mantenido en la edición inglesa de 2001 de la Northwestern University Press como Zollikon Seminars- citaremos aquí por la reciente edición francesa de Gallimard intitulada Séminaires de Zurich.
} 
ORIGINALES Y REVISIONES

no sólo relacionar (en uno u otro orden) realidades e ideas. La verdad se hallará en la existencia misma y no en una esencia oculta necesitada de complejos $-\mathrm{y}$ vistosos- procesos de intelección.

Partícipe de esta convicción, el saber fenomenológico asume la identificación entre las cosas y su mostrarse. En él las cosas son lo que parecen, se dan con una "fidelidad radical" (5) que permite al saber limitarse -en palabras de Heidegger- a "hacer ver desde sí mismo aquello que se muestra" (10). Desde sí mismo, sin escáner ni diván, sin precisar una sub-stancia o sub-jeto hipostasiados, un substrato neuronal (que no niega) o un subconsciente. La psiquiatría existencial-frente a los modelos tradicionales- no entenderá lo que aparece, se muestra o anuncia, el dolor, desde algo que no se muestra, sea por su localización intracraneal o inconsciente. Se centrará en el dolor mismo.

\section{El saber hacer psiquiátrico}

Atento al dolor, al mostrarse del mismísimo sufrimiento, el psiquiatra fenomenólogo hubo de reconocer, con todo, que el resultado de su exploración recaía en un científico sujeto reflexivo que "transforma las vivencias vividas en vivencias miradas, [que] habla sobre ellas y no desde ellas" (5). Un sujeto que, a pesar de su vocación de inmediatez, se halla atrapado en una refinada epistemología que lo acerca a la vida sin permitirle nunca alcanzarla. No se trata sólo de la "tensión esquematizante" que Ramos y Rejón (14) denuncian en Minkowski, no es el literal tomar conciencia de la distancia entre la estructura totalizadora del conocer y la concreción del síntoma, no es que el conocimiento que podamos obtener sea limitado o impreciso. La cuestión es que sea conocimiento. Lo problemático es que la relación terapéutica sea (o se fundamente en) una relación de conocimiento. Que el encuentro sea entendido, a lo sumo, como un preconocer.

Por eso la psiquiatría existencial (insatisfecha con una síntesis pasiva que no dejaba de ser otro engranaje de la máquina experiencial ${ }^{3}$ ) apela a esa persona que, si bien puede actuar como sujeto de conocimiento, rebasa esta función que pretende encasillarlo. Orilla esa conciencia ajena al mundo, por depurada y casi transparente que se la quiera, para volver a él. Porque la tensión del acto cognoscitivo - por muy distendido que sea- nunca podrá dar cuenta de la historia del

\footnotetext{
${ }^{3}$ Sobre la «síntesis pasiva» de Husserl, las «micropercepciones inconscientes» de Leibniz o la "«existencia indeterminada» que Kant detectaba en el «sentimiento de sí mismo» de Descartes" apunta José Luis Pardo: "todas estas perplejidades se producen en el seno de la filosofía de la conciencia" (Pardo JL. El sujeto inevitable. En Cruz (15), pp. 138-9).
} 
doliente: por ser la relación efímera y de conocimiento, obscena y fugaz, presta a la actuación de la histeria o la concesión de la psicosis: "oigo voces", para cerrar lo antes posible el lugar del encuentro, de la enunciación. La comprensión existencial reclama, frente a la consulta minutada, un "«saber» del mundo indistinguible de nuestro propio estar en él" (5). Un estar (o living) dilatado en el tiempo, dispuesto a una aprehensión de la vida que convenga a su gestación temporal, histórica. La actitud existencial consistirá, frente a la fenomenológica búsqueda de evidencias, frente a la indiscreción de la mirada escrutadora, en un costoso "saber estar", en un savoir faire pacientemente ejercitado.

\section{Las buenas intencionalidades}

Este ponderado paso atrás (o giro hermenéutico) por el que "dejar ser" responde a una renovada comprensión de la verdad. Supone, lejos de la aparente desidia, un arduo esfuerzo de reinterpretación histórica. Implica la dolorosa renuncia a una instancia última de legitimación, a la ansiolítica correspondencia (adaequatio) entre lo que se muestra a la conciencia y lo que se subpone que es. Apuesta por un concepto de verdad como revelación (alêtheia) que antepone la escucha a la insolente mirada, que no dirige los ojos sino que ofrece el oído, que no es sólo apremiado conocer objetivante, sino un tiempo para estar y tratar con la persona.

Sin embargo, no parece haberlo tomado en cuenta el contemporáneo análisis existencial, ajeno a unos desarrollos hermenéuticos que no integra aunque sí recoge. Martin Adams reconoce que las cosas mismas que Husserl considera como "estructuras esenciales de la conciencia trascendental" son desechadas en Heidegger como "residuos metafísicos, artefactos de una investigación fallida" (16). En la misma línea refiere Mick Cooper que los representantes de la perspectiva existencial, frente a la fenomenológica, rechazan la necesidad de una alambicada "conciencia pura y ego trascendental" y prefieren atender la existencia humana ya "inmersa en su mundo" (17). Este alejamiento de la conciencia trascendental, esta predilección por lo mundano, responde a la sorpresa del sujeto que piensa un mundo... en el que está ya inmerso. La "estructura esencial de la conciencia" que le sirve para entender el mundo no le basta al sujeto para comprender su previo estar en él. La conciencia se reconoce como actor secundario, como (a)parte, como el rezagado narrar de una historia ya empezada.

Esta cuestionada trascendentalidad o desfase, aceptada sobre el papel, permanece sin embargo incólume en Emmy Van Deurzen, adalid de la Escuela Británica de Análisis Existencial, quien se muestra "maravillada" (18) ante la paradoja del saberse retrasado, de la reconocida ficción, ante unos personajes cartón piedra. El 
ORIGINALES Y REVISIONES

errar de la holandesa sólo es comprensible si se toma por meta cualquier fin de camino, desvarío procurado por una lectura de la fenomenología $(16,17,18,19)$ que propone una descripción de la experiencia basada en la mera oposición a un explicar satanizado, biologicista y medicalizador. Esta escasa problematización del concepto verdad (y su método) redunda en un ejercicio de "interpretación" asaz confuso. Así, el Análisis existencial de Gion Fidel Condrau, presidente de la International Federation of Daseinsanalysis, aboga por una fenomenología que no trate la "conciencia sino el insight, lo que significa que se abstiene de interpretaciones y deja hablar a los fenómenos mismos" (20). Confiados en la puesta entre paréntesis o bracketing, sin tomar en consideración los desarrollos fenomenológicos que mostraron la necesidad de (re)interpretar siempre para volver a las cosas mismas, se termina fascinado como Van Deurzen (18) por un sujeto "transparente", por la gracia del insight. La ingenua demarcación de las posibilidades del describir y del acceso a la realidad terminará por simplificar la solución de un problema incomprendido. Si no podemos entender el mundo (porque vivirlo no se contempla todavía) habrá que dotarlo de un sentido. Esa es la tarea encomendada a la dimensión espiritual que Van Deurzen propone como supra-mundo (Überwelt), como sistema de significados (system of meaning) organizador y contextualizador de la experiencia ${ }^{4}$. Mas con esta dación, creación o búsqueda de sentido se procede no más que a una fútil tematización del mundo, con barniz existencial si se quiere pero de firme armazón narrativo (8). Se cree ingenuamente poder resolver por ensalmo la relación yo-mundo fracturada. Bastaría encontrar un sentido, construir una verdad aglutinadora o emplear ese "mero conector" (18), la mente, para restar trascendencia a la ruptura; bastaría aplicar el espeso bálsamo intencional de la conciencia para ocultar una herida que en la curación muestra su alcance: escuece en ella, como había advertido el mismo Husserl (y retomó Merleau-Ponty), la diferencia intencional.

A pesar de enfatizar el carácter problemático de esta lacerante diferencia yomundo, el análisis existencial -lejos de pensar un modo de acercar sus bordesalienta un inflamado plastrón. Con la mejor intencionalidad, abulta la cesura trascendental de una relación enfrentada y escasamente terapéutica.

\footnotetext{
${ }^{4}$ Si también Giovanni Stanghellini $(21,22)$ apela a unos organizadores de sentido es muy otro, a nuestro entender, el nivel de autocomprensión que los legitima.
} 


\section{Un lugar para el encuentro: entre la psicopatología y la terapia}

Resulta palpable (en la solidez de una mesa o la tersura de una bata) la distancia que separa al sujeto del saber, el médico, del paciente objeto. Esa va a ser la escisión fundamental que denunciemos en la práctica psiquiátrica: la violenta relación del conocer. Pero analizaremos ahora otro nivel más delicado, más difícil de conjurar con los epítetos "persona" o "cliente", con el furor de la esquiva empatía. Denunciaremos no el hachazo sino la carcoma del análisis existencial contemporáneo. No la herida sangrante sino la casi imperceptible fisura. Analizaremos el síntoma, sumido él también -como la relación- en la doblez de su mostrarse.

En ningún caso impugnamos los valiosos hallazgos psicopatológicos de la fenomenología psiquiátrica $(23,24)$. Sólo queremos constatar su confesa permanencia en una filosofía de la conciencia (25) de reconocida esterilidad terapéutica, desde Binswanger y Minkowski hasta la actualidad (15, 26, 27, 28): "no hay una "psicoterapia fenomenológica" reconoce Fuchs (26), mientras Stanghellini y Lysaker conceden que las implicaciones para la psicoterapia de sus investigaciones sobre la conciencia "deben aun ser probadas" (22). Por el momento la tarea fenomenológica se ha ceñido al análisis exhaustivo, a la exploración detallada y a la consiguiente reelaboración descriptivo-constructiv(ist)a $(19,22,26,29)$. A la reconstrucción de la experiencia, a la pulimentación psicopatológica (11).

Enterada de sus limitaciones, la fenomenología se centrará en despejar el escenario de la descripción. Cauta ante una intervención precariamente articulada, atiende el claro de la relación terapéutica. Se esfuerza en la apertura de un espacio prejudicativo, un ámbito de encuentro independiente de su posterior uso psicopatológico, entendido no tanto como momento pasivo o prepredicativo de construcción de la experiencia sino como una negatividad (y no prepositividad) en la que dicho encuentro pueda darse. No tanto como el conocimiento tácito (tacit knowledge), todo lo inarticulado y preconceptual que se quiera, de Mahoney; sino más bien como el sentimiento implícito (felt implicit) de Gendlin (8). Un estar previo al saber, un "hacer hueco" $(26,30)$ que permita entrar en relación antes del pensar psicopatológico. La cuestión es si dicha apertura puede entenderse libre de la tensión cognoscitiva, si puede obviar la expectación del saber y contener la compulsión epistémica. Si en lugar de prealgo puede entenderse como espacio propio, con valor por sí. Espacio no sólo abierto a la analítica captura o aprehensión del otro sino dispuesto a un encuentro en el que compartir $s u$ mundo, $s u$ horizonte. 
ORIGINALES Y REVISIONES

Fisuras del análisis existencial contemporáneo: dos ejemplos clínicos

Ajeno al fino proceder de la psicopatología fenomenológica mentada; extraño al reconocimiento de sus propios límites, al saberse la fragilidad y el riesgo, el análisis existencial ha suturado por lo sano sin atender la fisura que lo mina: una psicopatología trascendental arrebatada, incapaz de sostener unas propuestas terapéuticas que colapsarán sin remedio la siempre volátil aparición del dolor $(31,32)$.

\section{Hipocondría}

Alice Holzhey-Kunz, presidenta de la Sociedad Suiza de Análisis Existencial, aproximando hermenéutica y psicoanálisis pone en juego una forma de interpretación asentada en la metafísica de las esencias: "los fenómenos existenciales están escondidos", afirma, y habrá por tanto que descubrirlos. Esta manera de "interpretar" nada tiene que ver con la Auslegung ${ }^{6}$ heideggeriana, que Gianni Vattimo describe como "articulación de una comprensión originaria en la cual las cosas están ya descubiertas" (33). Toma Holzhey-Kunz como ejemplo la hipocondría y dice que en ella "cada síntoma corporal, por leve que sea, se refiere no sólo a la fragilidad de nuestro cuerpo, sino al hecho de que nunca podemos tener la certeza de no estar afectados por una severa y eventualmente mortal enfermedad". Se refiere (el destacado era nuestro), alude o se corresponde... con algo que no se muestra, con una esencia hipostasiada. Volvemos al homo psychologicus, al sujeto escindido pendiente de ser descifrado (34). Reasumida la tópica división en el sujeto, ya poco importa que las relaciones o dinámicas se den en una tematización ("contexto oculto") psicoanalítica o existencial. Según este modelo dinámico (de correspondencias), hay que atender la relación entre el miedo abstracto a morir (facticidad ontológica) y el temor palpable a enfermar, a una fatal pleuresía o a la oclusión intestinal (hecho óntico). Se apela, lo mismo da, a una supraestructura o un subconsciente para dotar al mundo de un sentido que, mal nos pese, lo tiene ya (y al descubierto). Este proceso por el que identificar un correlato ontológico (o inconsciente) de los hechos, más allá de la escasa legalidad ya advertida por Heidegger para la cura, se torna un proceder de cínica esterilidad, pues conduce -como reconoce la misma Holzhey-Kunz- a una "tarea cuya compleción rebasa los objetivos de la terapia analítica. El paciente deberá encontrar las respuestas por sí mismo y responsabilizarse de ellas -el analista no puede guiarlo en esto- pues de otro modo no estaría respetando la autonomía de su paciente". No es la tarea de compleción (endosada al enfermo) lo que flaquea. Son los mismos principios

\footnotetext{
${ }^{5}$ Holzhey-Kunz A. What defines the Daseinsanalytic process? En Du Plock (9), pp. 200-10

${ }^{6}$ Stanghellini (29) opone Auslegung o explication a Erklärung o explanation, en la que se apela a mecanismos sub o transpersonales del saber.
} 
de esta "terapia analítica", de esta "interpretación" propuesta por el terapeuta, los que obstaculizan el comprender, los que expulsan la verdad más allá de las cosas mismas. Entonando el ritornelo de las esencias, nos remiten a instancias de otro nivel, nos embarcan (solos) rumbo a causalidades extrañas, sean las preocupaciones últimas (ultimate concerns) de Yalom o los traumas psicosexuales freudianos. El mecanismo propuesto para la curación es conocido: la tosca y denostada explicación (explanation). Aceptado esto, "sobre qué base -se pregunta Cooper y nosotros con él- puede un psicoterapeuta existencial-fenomenológico privilegiar el discurso de las 'experiencias vividas' o 'preocupaciones ontológicas' sobre otros discursos terapéuticos, como el de los 'procesos inconscientes' o ‘impulsos libidinales'?”7

\section{Disociación}

Vista la necesidad del dolor de remitirse siempre a un referente oculto para su comprensión, veamos ahora cómo el yo mundanizado, viviente (y doliente), diverso, reclama un titiritero que lo sostenga. Constatamos la furibunda renuencia del subjeto a dejar ver "aquello que se muestra", entremetiéndose siempre. Tomamos como ejemplo para ello la "reinterpretación fenomenológica" que Ernesto Spinelli hace de la disociación como polipsiquismo. Presentación psicopatológica sin igual para destacar la diversidad del existir, nos hace recular de nuevo hacia la homogeneización del sujeto. Se concibe -en resumen de Cooper- la disociación como un "modo fenomenológico de encontrarse en el mundo al tiempo que se mantiene un "auto-constructo sedimentado"". Se abrocha, temiendo el desparrame, el corsé unificador. Se cree precisar un "sedimento" para sostener una existencia plural. Escuchamos y vemos a la persona llorosa, angustiada, corpórea; mientras pensamos un sujeto impasible, ideal y silencioso. El mismo Spinelli reconoce que el sujeto "que postulamos como proceso relacional lo experimentamos ingenuamente desde una postura esencialista" . ¿Por qué invocamos siempre y lo primero un conocimiento que enturbia el "encontrarse en el mundo"? ¿Por qué permanecemos o nos precipitamos sin remedio en la categórica epistemología?

Sometidos al dictamen trascendental debemos reconocer -nuevamente- la esterilidad terapéutica de nuestro ingenuo (en palabras de Spinelli) proceder. No sólo se admite que "dicho foco fenomenológico-existencial no niega enteramente la existencia de entidades intrapsíquicas de ubicación estructural -sino que, como admite Cooper-, minimiza notoriamente su posible significado terapéutico". No sólo recaemos en la metafísica sustancial (hasta eso llegar Cooper queriendo evitarlo) sino que con ello se entorpece, y es aquí lo que importa, la tarea terapéutica.

\footnotetext{
${ }^{7}$ Cooper M. The Discourse of Existence. Existential-Phenomenological Psychotherapy in a Postmodern World. En Du Plock (9), pp. 392-9

${ }^{8}$ Cooper M. Modes of Existence. Towards a Phenomenological Polypsychism. En Du Plock (9), pp. 147-53

9 Spinelli E. The Vagaries of the Self. En Du Plock (9), pp. 189-99
} 
ORIGINALES Y REVISIONES

\section{Conclusión}

La relación terapéutica no puede ser entendida como una relación de conocimiento. Escapar, huir, o intentarlo, del paradigma de la conciencia será necesario para establecer una relación con el doliente no limitada a un mirar taxonómico. Si tras superar la sustancialización del mundo antiguo creíamos avanzar con la moderna subjetividad kantiana, con el sujeto de conocimiento, la hermenéutica nos despierta del engaño: hemos pasado del fuego de la sustancia a las brasas de la conciencia. La persona continúa sin ser tal y debe apoyarse siempre en un universal reconocible, sea sustancia en la cosa o conciencia en el sujeto (35). El fantasma de la esencia y las cadenas del pensamiento representacional sancionan una servidumbre que ha frustrado la verdadera relación personal, o intersubjetiva. Una cerrazón que no ha permitido tratar con la persona sino del sujeto (36).

Heidegger quiso hacer desaparecer esa moderna subjetividad (5) que adolecía de los mismos vicios objetualizadores de la sustancia. Si muchos quisieron creer que lo había logrado, muestra sus reticencias el ultramoderno Jürgen Habermas. Según él la denostada "primacía ontológico/epistemológica" (37) que Heidegger pretende rehuir atrapa también a su analítica del Dasein. Si atendemos la ya mentada deuda de Heidegger con la fenomenología husserliana, no sorprende que Habermas le pueda reconvenir sus intentos de superación hermenéutica de la razón trascendental. Pero como Heidegger no sigue únicamente la estela de Husserl, debe reconocer el autor de El discurso filosófico de la modernidad que a su crítica escapan los motivos de la filosofía de la existencia insertados en la obra del de Messkirch. "Impulsos me dio Kierkegaard y ojos me los puso Husserl" reconocía el mismo Heidegger ${ }^{10}$. El impulso kierkegaardiano (38) sobrevuela los escollos de la modernidad. Si el camino fenomenológico se torna intransitable más allá de la lisa relación del conocer (5), se halla en la filosofía de la existencia de Kierkegaard la porosidad, oquedad o resquicio por donde escapar del vasallaje epistémico.

La obra de Heidegger, entendida con Günter Figal como "corroboración fundamental-ontológica del diagnóstico kierkegaardiano” (39), posibilita el establecimiento de una relación verdaderamente terapéutica y no de mero conocimiento. Huidos de "la primacía metodológica de la autorreflexión" (37) que lastra a la fenomenología, nos permite ocupar y definir un nuevo espacio de encuentro, abierto al horizonte, distinto de la asfixiante antesala del conocer. La conquista de esta negatividad, de este lugar que nada presupone, que no es -en ningún sentido- prepositivo sino mera apertura, claridad, es lo que distinguiría una propuesta terapéutica verdaderamente existencial.

\footnotetext{
${ }_{10}$ Rodríguez (5, p.94) nos recuerda que con "estas palabras abría Heidegger en 1923 el curso Ontología. Hermenéutica de la facticidad, primer anticipo sistemático de Ser y tiempo".
} 
La fenomenología, en su legítimo afán estructurador del conocimiento, acarrea siempre un sujeto de la conciencia. El proyecto heideggeriano no solo consiste en sustituir ese Yo trascendental por el Dasein, sino que en sus desarrollos tardíos se desliza del hombre a un espacio dispuesto para el acaecimiento-apropiación (Ereignis) del Ser (40). No se trata de proponer una nueva subjetividad o cerrar el catálogo de sus restos, sino de abrir un nuevo ámbito de relación.

La incansable repetición de la pregunta por la subjetividad o el ser nos sorprende, ya no en la voz de un sujeto, sino en el eco de un lugar. Querríamos definir y abrazar al dueño de ese verbo emancipado, pero la rugosidad de la caverna nos despista y la corporeidad del sonido destruye su inmediatez. Ante lo grotesco no apelaremos a la razón. Resistiremos a la persecutoria metafísica. Resultará de esta actitud una sutil pero tajante distinción: un nuevo modo de ser, estar y comportarse. Se prestará atención a una hondura del pensar que no es cimiento sino abismo, irracionalidad que exige un salto. A una profundidad radical que no luce como la interpretación o la magnética resonancia. Una implicación que permanecerá ínter nos, en el trato espacial y silencioso. Un saber estar que no podrá -como nos advierte Heidegger- "ser distinguido desde fuera en absoluto" (11). Mas sí en la relación, por cada uno dentro.

BIBLIOGRAFÍA:

(1) Ramos P. El desenvolvimiento de la psicopatología, Archivos de Psiquiatría 2000; 63(4): $327-338$

(2) Villegas M. La psicología humanista: historia, concepto y método. Anuario de psicología 1986; 34(1): 7-45

(3) Heidegger M. Carta sobre el humanismo. Madrid: Alianza Editorial, 2000

(4) Burston D. Existentialism, Humanism and Psychotherapy. Existential Analysis 2003; 14(2): $309-19$

(5) Rodríguez R. Hermenéutica y subjetividad. Madrid: Trotta, 2010

(6) Yalom I. Psicoterapia existencial y terapia de grupo. Barcelona: Paidós, 2000

(7) Diéguez A. Neopragmatismo y posmodernidad en psicoterapia. Rev Asoc Esp Neuropsiq 2008; 28(101): 147-64

(8) Richert AJ. Some Thoughts on the Integration of Narrative and Humanistic/Existential Approaches to Psychotherapy. Journal of Psychotherapy Integration 1999; 9(2): 161-84

(9) Du Plock S, editor. Further Existential Challenges to Psychotherapeutic Theory and Practice. London: The Society for Existential Analysis, 2002

(10) Heidegger M. Ser y tiempo. Madrid: Trotta, 2009

(11) Heidegger M. Séminaires de Zurich. Paris: Gallimard, 2010

(12) Diéguez A. Richard Rorty: La inquietante huella de Nietzsche. Frenia 2006; VI: 219-25

(13) Tillich P. Existential Philosophy. Journal of the History of Ideas 1944; 5(1): 44-70 


\section{ORIGINALES Y REVISIONES}

(14) Ramos P, Rejón C. Los síntomas de la psicopatología. Identificación e interpretación. Actas Esp Psiquiatr 2002; 30(4): 213-20

(15) Cruz M, compilador. Tiempo de subjetividad. Barcelona: Paidós, 1996

(16) Adams M. Practising Phenomenology, some reflections and considerations. Existential Analysis 2001; 12(1): 65-84

(17) Cooper M. Existential Therapies. London: SAGE publications, 2008

(18) Van Deurzen E. Everyday Mysteries. Existential Dimensions of Psychotherapy. East Sussex: Routledge, 2009

(19) Carvalho Teixeira J.A. Introdução à psicoterapia existencial. Análise Psicológica 2006; 3(24): 289-309

(20) Condrau GF. Daseinsanalytic Psychotherapy. [consultado 27 de enero de 2011]: Disponible en: $\quad$ http://www.daseinsanalyse.com/ifda/daseinsanalysis.html

(21) Stanghellini G, Ballerini M. Values in Persons With Schizophrenia. Schizophr Bull 2007; 33(1): 131-41

(22) Stanghellini G. y Lysaker PH. The psychotherapy of schizophrenia through the lens of phenomenology: intersubjectivity and the search for the recovery of first- and second-person awareness. Am J Psychother 2007; 61(2): 163-79

(23) Parnas J, Sass LA, Zahavi D. Recent developments in philosophy of psychopathology. Curr Opin Psychiatry 2008; 21(6): 578-84. Review

(24) Owen G, Harland R, editors. Phenomenology and Psychiatry for the 21st Century [tema especial]. Schizophr Bull 2007; 33(1): 105-65. Disponible en http://schizophreniabulletin.oxfordjournals. org/content $/ 33 / 1$. toc

(25) Novella EJ, Huertas R. El Síndrome de Kraepelin-Bleuler-Schneider y la Conciencia Moderna: Una Aproximación a la Historia de la Esquizofrenia. Clínica y Salud 2010; 21(3): 205-19

(26) Fuchs T. Psychotherapy of the lived space: a phenomenological and ecological concept. Am J Psychother 2007; 61(4): 423-39

(27) Cohn H. What is Existential Psychotherapy? Br J Psychiatry 1994; 165: 699-701

(28) Villegas M. Ludwig Binswanger: En el centenario de su nacimiento. Anuario de psicología 1981; 24(1): 5-26

(29) Stanghellini G. A Hermeneutic Framework for Psychopathology. Psychopathology 2010; 43: $319-26$

(30) Ramos P. Vida, psiquiatría y biopolítica. Un asunto psicopatológico, Frenia 2009; IX: 7-31

(31) Martínez Hernáez A. Antropología versus psiquiatría: el síntoma y sus interpretaciones. Rev Asoc Esp Neuropsiq 1998; 18(68): 645-59

(32) Martínez Hernáez A. Cuando las hormigas corretean por el cerebro: retos y realidades de la psiquiatría cultural. Cad. Saúde Pública, Rio de Janeiro 2006; 22(11): 2269-2280. Revisão

(33) Vattimo G. Introducción a Heidegger. Barcelona: Gedisa, 2000

(34) Novella EJ. Foucault, la psicoanàlisi i el subjecte. Quaderns de filosofia i ciència 2007; 37: 29-38

(35) Gadamer H.-G. El giro hermenéutico. Madrid: Cátedra, 2001

(36) Colina F. Actualidad hermenéutica de las psicosis. Frenia 2002; II: 109-19

(37) Habermas J. El discurso filosófico de la modernidad. Madrid: Katz, 2008

(38) Kierkegaard SA. La enfermedad mortal. Madrid: Trotta, 2008 
(39) Figal G. Indiferenciación vital y distanciamiento. La actitud hacia sí en relación con Heidegger, Kierkegaard y Hegel. Buenos Aires: Biblos, 2010

(40) Volpi F. Martin Heidegger. Aportes a la filosofía. Madrid: Maia, 2010 